\title{
Lactococcus garvieae
}

National Cancer Institute

\section{Source}

National Cancer Institute. Lactococcus garvieae. NCI Thesaurus. Code C86484.

A species of facultatively anaerobic, Gram positive, cocci shaped bacteria assigned to the phylum Firmicutes. This species is nonmotile, catalase and cytochrome oxidase negative, nonsporulating, and alpha hemolytic on blood agar. L garvieae is a zoonotic pathogen, especially in fish. 